ReVISTA INTERnaCIONAL de SOCIOLOGÍa (RIS)

Vol.68, N³, Septiembre-DicIEMBRE, 775-795, 2010

ISSN: 0034-9712

elSSN: 1988-429X

DOl:10.3989/ris.2008.10.29

\title{
LA PERCEPCIÓN DE LOS TRABAJADORES DE LA NORMATIVA DEL SISTEMA PÚBLICO DE PENSIONES El caso español
}

\author{
WORKERS' PERCEPTION OF THE PUBLIC SYSTEM OF PENSIONS \\ The Case of Spain
}

Ramón Cobo-Reyes

Universidad de Granada. España

ramonc@ugr.es

JuLIÁN Díaz-SAaVedRa

Universidad de Granada. España

julianalbertodiaz@ugr.es

\begin{abstract}
REsumen
Durante las últimas décadas, la edad media de jubilación de los trabajadores en los países desarrollados se ha reducido notablemente. Una cuestión discutida, sin embargo, es si esta conducta de jubilación es consecuencia o no de la normativa que rige los sistemas públicos de pensiones. Si esta conducta es consecuencia principalmente de dicha normativa esto indica que los trabajadores la conocen y la comprenden. Caso contrario, entonces los trabajadores no la conocen, o la conocen pero no la comprenden. En este trabajo, analizamos la normativa del Sistema Público de Pensiones en España, y basándonos en ella, obtenemos una conducta teórica óptima de jubilación, dadas las características individuales del trabajador. Posteriormente, analizamos la conducta empírica de jubilación de los trabajadores en España. Encontramos que esta conducta empírica es similar a la conducta teórica, con lo cual concluimos que los trabajadores de edad avanzada sí conocen y entienden la normativa del Sistema Público de Pensiones español.
\end{abstract}

\section{Palabras Clave}

Conducta de Jubilación, Comprensión, Protección Social.

\begin{abstract}
During the last years, the mean retirement age in developed countries has decreased. An important question is if this retirement behavior is a consequence or not of the law applied to pensions. If the behavior is a consequence of this law, then this means that workers know and understand the law. On the other side, workers do not know the law, or they know it but they do not understand it. This paper studies the law applied to the Public Pension System in Spain and obtains workers' optimal retirement behavior, given some workers' features. Then, we analyze the retirement behavior of workers in Spain. We find that the retirement behavior of workers in Spain is very similar to that at the optimal retirement behavior predicted by theory, so we conclude that Spanish workers know and understand the law.
\end{abstract}

\section{KEYWORDS}

Retirement Behaviour Jubilación, Social Insurance, Understanding. 


\section{INTRODUCCIÓN}

La sostenibilidad financiera de los sistemas públicos de pensiones de la mayoría de los países industrializados comienza a verse cuestionada por dos factores principales. En primer lugar, debido al proceso de envejecimiento poblacional. Y en segundo lugar, por la tendencia de los trabajadores a jubilarse en edades más tempranas. Consecuentemente, la reforma del sistema de seguridad social es uno de los principales puntos en las agendas de los gobiernos de estos países.

Distintas son las reformas que han sido propuestas, con el fin de mejorar la viabilidad financiera futura de estos sistemas de protección social, como por ejemplo aumentos en las tasas de contribución o incluso la reducción en las prestaciones por jubilación. Además, y con el fin de revertir la tendencia hacia la jubilación anticipada, se ha propuesto el aumento en la edad de jubilación (Boldrin y Jiménez-Martín 2002) o un cambio en la normativa de dichos sistemas con el objeto de desincentivar la jubilación anticipada (Lacomba y Lagos 2007; Fatás, Lacomba y Lagos 2007).

A la hora de tomar las decisiones sobre la jubilación, la literatura especializada distingue dos tipos de información que necesitan los trabajadores para poder desarrollar sus planes. Por un lado necesitan información de carácter técnico (conocimiento sobre inversiones, leyes, cobertura del sistema médico, etc.) y por otro lado los individuos también necesitan cierta información sobre su propia salud y potencial de renta.

Una cuestión discutida es si la decisión de la edad de jubilación por parte de los trabajadores es consecuencia, principalmente, de la normativa que rige tales sistemas. Existen dos visiones opuestas acerca de esta cuestión. La primera de ellas sostiene que la conducta de jubilación de los trabajadores no se debe a la normativa que rige los sistemas públicos de pensiones y sí a otros factores, como la salud y riqueza de los mismos. Prueba de ello, según este enfoque, es que la evidencia demuestra que los trabajadores no entienden e incluso desconocen la normativa de dichos sistemas. Por lo tanto, si un trabajador no conoce estas reglas, los sistemas de pensiones no pueden afectar su conducta de jubilación. Por ejemplo, en un análisis basado en el Survey of Consumers Finances, Mitchell (1998) encuentra que los trabajadores adultos en Estados Unidos tenían una gran falta de información acerca de las provisiones en una jubilación anticipada. Otros autores como Juster and Suzman (1995), también para el caso de Estados Unidos, en su obra Health and Retirement Study concluyen que, generalmente, los sujetos no tienen un gran conocimiento acerca de la estructura de incentivos que tienen sus planes de pensiones privados.

Otros estudios, sin embargo, sostienen que si bien variables como la salud y la riqueza del trabajador influyen en su decisión de jubilación, el elemento principal que determina esta conducta se encuentra últimamente en los sistemas públicos de pensiones. A esta conclusión llegan Mahieu y Blanchet (2004) quienes analizan la conducta de jubilación de los trabajadores en Francia, Wise (1997) que analiza esta conducta en 
Estados Unidos, y Börsch-Supan et al. (2002) en el caso de los trabajadores en Alemania'.

La obtención de una conclusión acerca de si los trabajadores entienden o no las reglas del Sistema de la Seguridad Social se torna de gran relevancia. Por un lado, a la hora de proponer reformas para mejorar la viabilidad financiera futura de dichos sistemas, conocer el nivel de comprensión que los trabajadores tienen de la normativa puede ayudar de una forma crucial a la obtención de resultados más eficaces, a través de su impacto en la conducta de jubilación. Por otro lado, y relacionado con lo anterior, esto podría validar o no los modelos económicos utilizados para evaluar cualitativamente y/o cuantitativamente, el impacto de las reformas, debido a que estos modelos asumen, en general, que los trabajadores desconocen la normativa².

Este trabajo trata de aportar más evidencia con el fin de dilucidar esta cuestión. Específicamente, intentamos responder a la pregunta de si los trabajadores en España conocen y comprenden la normativa que rige el Sistema Público Español de Pensiones. Para responder a esta pregunta realizamos el siguiente análisis: primero, analizamos la normativa del principal régimen que compone el sistema de pensiones en España, el Régimen General de la Seguridad Social. Posteriormente, y basándonos en dichas normas, obtenemos una conducta teórica óptima de jubilación, dadas las características individuales del trabajador. Por último, comparamos esta conducta con la conducta empírica de jubilación de los trabajadores en España. Nuestros resultados muestran que ambas conductas son similares, por lo que deducimos que los trabajadores de edad avanzada sí conocen y comprenden las normas que establece el Régimen General de la Seguridad Social.

Este trabajo está organizado de la siguiente forma. En la sección 2 describimos la normativa del Régimen General de la Seguridad Social. En la sección 3 determinamos cuál debería ser la conducta óptima de jubilación, en función de dicha normativa y de las características del trabajador. La sección 4 describe la conducta empírica de jubilación de los trabajadores en España, y la compara con la conducta óptima de jubilación encontrada en la sección anterior. Finalmente, la sección 5 expone nuestras principales conclusiones.

\section{El Sistema Público Español de Pensiones}

El origen del actual Sistema Público Español de Pensiones data de 1900 con la creación del Seguro Obligatorio para empleados públicos, que otorgaba a estos pensiones por

\footnotetext{
${ }^{1}$ Para alcanzar esta conclusión, estos trabajos analizan empíricamente la conducta de jubilación de los trabajadores, para posteriormente racionalizar dicha conducta en función de los incentivos implícitos que proveen los sistemas públicos de jubilación, a través de su normativa.

${ }^{2}$ Notables excepciones son los trabajos de Feldstein (1977), Sheshinski (1978) o Kotlikoff (1979).
} 
jubilación y por incapacidad derivada de accidentes de trabajo. Desde entonces, se han ido creando paulatinamente nuevos regímenes con el fin de aumentar el número de personas con derecho a una pensión de jubilación, incapacidad o demás contingencias susceptibles de ser protegidas por el Estado. En 1979 se crea el Instituto Nacional de la Seguridad Social, organismo público que administra el Régimen General de la Seguridad Social y demás regímenes especiales que conforman el actual Sistema Público Español de Pensiones.

En esta sección describimos las principales normas del Régimen General de la Seguridad Social, el más importante de España en cuanto a número de afiliados ${ }^{3}$. La normativa que describiremos corresponde al sistema vigente entre los años 1994 y 1999, y esto se debe a que los datos de los que disponemos para realizar nuestro análisis acerca de la conducta empírica de la jubilación se refieren precisamente a ese mismo período.

\section{Financiación}

El Régimen General de la Seguridad Social se financia principalmente a través de las cotizaciones de sus afiliados. La cotización de un trabajador se calcula a través de la aplicación de un tipo de cotización fijo sobre la base de cotización, siendo ésta la renta salarial mensual del trabajador, entre un mínimo y un máximo exento. El tipo de cotización es actualmente el 28,3 por ciento (29,3 por ciento hasta enero de 1995), del cual el 23,6 por ciento es pagado por la empresa, mientras que el 4,7 restante recae sobre el trabajador. En la actualidad, la recaudación total por cotizaciones en España es aproximadamente del 10 por ciento del PIB, según los datos del Ministerio de Trabajo y Asuntos Sociales ${ }^{4}$.

\section{Gastos}

Debido a que el Régimen General de la Seguridad Social es un sistema de reparto, la recaudación total por cotizaciones, más la aportación que pudiera realizar el Estado, se utiliza para el pago de las prestaciones totales que provee este régimen. En la actualidad, de la cuantía total de prestaciones pagadas por este régimen, el 67 por ciento corresponde al pago de pensiones de jubilación, y el 33 por ciento restante corresponde al pago de pensiones por incapacidad, orfandad y demás contingencias ${ }^{5}$. A continuación describimos las principales normas que regulan el derecho para acceder a una pensión de jubilación.

\footnotetext{
${ }^{3}$ En el año 2001 más del 73 por ciento de los trabajadores estaban afiliados en España a este régimen.

${ }^{4}$ Estos datos están disponibles en http://www.mtas.es/estadisticas/anuario.htm

${ }^{5}$ Memoria 2006 del Instituto Nacional de Seguridad Social. Esta memoria está disponible en http://www. seg-social.es/Internet,/Estadistica/Documentacion/Memorias/index.htm
} 


\section{Elegibilidad}

Para tener derecho a una pensión de jubilación se requiere que el trabajador tenga un mínimo de 15 años cotizados, de los cuales dos de ellos deben estar dentro del período de ocho años inmediatamente antes de su jubilación.

\section{Pensión}

El componente principal de la pensión de jubilación es la Base Reguladora. Bajo el sistema vigente hasta 1997, ésta se define como la media de las bases de cotización durante los últimos 8 años anteriores a la fecha de jubilación. Las bases de cotización durante los dos años anteriores a la jubilación no se ajustan con la inflación. En los años anteriores sin embargo, se realiza un ajuste de forma tal que las bases de cotización se convierten al equivalente de la base del mes 25 antes de la jubilación. Sea $t$ el mes en el cual una persona decide jubilarse, y $P_{t-i}$ y $C_{t-i}$ la tasa de inflación y la base de cotización en el mes $i$ antes de la jubilación, respectivamente. Entonces, la Base Reguladora de una persona que decide jubilarse en el mes $t, R_{t}$ es:

$$
R_{t}=\frac{1}{112}\left\{\sum_{i=1}^{24} C_{t-i}+\sum_{i=25}^{96} C_{t-i} \frac{P_{t-25}}{P_{t-i}}\right\}
$$

donde $\sum_{i=1}^{24} C_{t-i}$. representa a las bases de cotización que no se ajustan con la inflación y $\sum_{i=25}^{96} C_{t-i} \frac{P_{t-25}}{P_{t-i}}$ son las bases de la cotización ajustadas.

A su vez, la Base Reguladora se multiplica por una tasa de sustitución, la cual depende del número total de años cotizados al Régimen General de la Seguridad Social. Sea $s$ el número de años cotizados por el trabajador durante su vida laboral. Bajo el sistema vigente hasta 1997, la tasa de sustitución, $\phi$, viene dada por la siguiente expresión:

$$
\phi= \begin{cases}0 & \text { si } s<15 \\ 0,6+0,02(s-15) & \text { si } 15 \leq s<35 \\ 1 & \text { si } 35 \leq s\end{cases}
$$

Esto implica que cuantos más años esté cotizando un individuo, mayor será la base reguladora ajustada por la tasa de sustitución.

En julio de 1997 se implementó una pequeña reforma sobre esta normativa, la cual consistió en dos cambios principalmente. Primero, se aumentó el número de años utili- 
zados para calcular la Base Reguladora, pasando desde los últimos 8 a los últimos 15 años inmediatamente anteriores a la jubilación. Por lo tanto, la nueva fórmula pasó a ser:

$$
R_{t}=\frac{1}{210}\left\{\sum_{i=1}^{24} C_{t-i}+\sum_{i=25}^{180} C_{t-i} \frac{P_{t-25}}{P_{t-i}}\right\}
$$

El segundo cambio afectó a la tasa de sustitución. A partir de julio de 1997 dicha tasa se calcula de la siguiente forma:

$$
\phi= \begin{cases}0 & \text { si } s<15 \\ 0,5+0,03(s-15) & \text { si } 15 \leq s<25 \\ 0,8+0,02(s-25) & \text { si } 25 \leq s<35 \\ 1 & \text { si } 35 \leq s\end{cases}
$$

Jubilación anticipada

Durante el período 1994 a 1999 la edad normal de jubilación era a los 65 años. Sin embargo, los trabajadores podían optar a la jubilación anticipada a partir de los 60 años si habían empezado a cotizar al Régimen General de la Seguridad Social antes del año $1967^{6}$. Si una persona optaba por jubilarse anticipadamente, su pensión de jubilación se reducía en un ocho por ciento por cada año anterior a los 65 . Sea $j$ la edad de una persona que decide jubilarse. Entonces, la penalización por jubilación anticipada de una persona con edad $j, \lambda_{j}$, se define como:

$$
\lambda_{j}= \begin{cases}0,4-0,08(j-60) & \text { si } j<65 \\ 0 & \text { si } j \leq 65\end{cases}
$$

Como se observa en la ecuación (5), la penalización por jubilación anticipada era de un 8 por ciento por cada año anterior a los 65 años. Esto implica entonces que si una persona decide jubilarse a los 60 años, la penalización máxima por jubilación

\footnotetext{
${ }^{6}$ En el año 2002, la edad mínima de jubilación se aumentó hasta los 61 años, pero continuaba siendo a
} los 60 años para aquellos trabajadores que cumplieran con algunos requisitos adicionales. 
anticipada sería del 40 por ciento, con lo cual recibiría el 60 por ciento de la cantidad resultante de multiplicar la Base Reguladora por la tasa de sustitución?

\section{Cuantías mínima y máxima}

Existe una cuantía mínima y una máxima sobre la pensión de jubilación, las cuales se legislan anualmente, y se las conocen como pensiones de jubilación mínima y máxima, respectivamente. Si la pensión de jubilación, calculada según las normas que se describieron anteriormente, cae por debajo de la cuantía mínima, la persona recibe un complemento hasta alcanzar la pensión mínima de jubilación. Si por el contrario, el cómputo de la pensión de jubilación excede la cuantía máxima, la persona que decida jubilarse sólo cobrará mensualmente la pensión máxima. Sean $\underline{b}$ y $\bar{b}$ la pensión mínima y máxima de jubilación, respectivamente. Entonces, la pensión de jubilación de una persona con edad $j$ que decida jubilarse en el mes $t, b_{j, t}$ debe cumplir:

$$
\underline{b} \leq b_{j, t} \leq \bar{b}
$$

donde, y de acuerdo a lo ya señalado, $b_{j, t}$ viene dada por la siguiente expresión:

$$
b_{j, t}=\left(1-\lambda_{j}\right) \phi R_{t}
$$

\section{LA CONDUCTA ÓPTIMA DE JUBILACIÓN}

Si el trabajador conoce y comprende las normas que acabamos de describir en la sección anterior, este puede planificar óptimamente su conducta óptima de jubilación, en función de sus características personales. Específicamente, desde la primera edad legal de jubilación, y dado el historial laboral pasado y presente de una persona, la normativa del sistema de pensiones en España genera beneficios y costes relacionados con la decisión de continuar trabajando un año más. Cuando el coste de seguir un año más trabajando sea superior al beneficio, decimos que la conducta óptima de jubilación es precisamente jubilarse en ese momento. Si por el contrario, los beneficios son superiores, entonces la conducta óptima de jubilación consiste en esperar al menos un año más antes de abandonar el mercado laboral y empezar a cobrar la pensión de jubilación.

Por lo tanto, procedemos primero a establecer cuáles son esos beneficios y costes que acabamos de mencionar, para después poder compararlos y determinar así la conducta óptima de jubilación, como función del historial laboral pasado y presente de una persona.

\footnotetext{
${ }^{7}$ La reforma de 1997 redujo esta penalización por jubilación anticipada del 8 al 7 por ciento anual, pero sólo para aquellos trabajadores que contaban con más de 40 años cotizados.
} 


\section{Costes y beneficios de continuar trabajando}

Supongamos que una persona ha alcanzado los 60 años y que debido a que ha cotizado al Régimen General de la Seguridad Social al menos 15 años tiene derecho a recibir una pensión de jubilación. En ese momento la persona debe decidir si sigue trabajando un año más o si, por el contrario, se jubila ${ }^{8}$. Dadas las reglas del Régimen General de la Seguridad Social, continuar trabajando un años más implica:

1. Se reduce la penalización por jubilación anticipada. Esto es así porque por cada año trabajado después de los 60 y antes de los 65 , esta penalización se reduce en 8 puntos porcentuales, como lo indica la expresión (5).

2. Puede aumentar su tasa de sustitución. Esto se cumple siempre y cuando la persona tuviera menos de 35 años cotizados, como lo indican las expresiones (2) y (4).

3. Cambia la Base Reguladora. Los ingresos salariales que recibirá la persona a lo largo del año, reemplazarán a los observados durante el primero de los 8 años anteriores, bajo la normativa vigente hasta 1997, como lo indica la expresión (1). Con la normativa vigente después de 1997, los ingresos salariales reemplazarán a los observados durante el primero de los 15 años anteriores, como lo indica la expresión (3).

4. El trabajador debe pagar al Régimen General de la Seguridad Social las cotizaciones derivadas de su renta salarial.

5. El trabajador no puede recibir la pensión de jubilación.

Independientemente del historial laboral pasado y presente de la persona, los puntos 1 y 2 se identifican claramente como beneficios generados a partir de la decisión de trabajar un año más, mientras que los puntos 4 y 5 son los costes que genera tal decisión.

En este punto es importante resaltar que la pensión mínima de jubilación está exenta de penalizaciones por jubilación anticipada. Esto significa que para un trabajador que debido, por ejemplo, a bajas rentas salariales pasadas, sólo pueda optar a este tipo de pensión, cobrará esta cuantía independientemente de la edad a la que se jubile. Nótese que el recibir este tipo de cuantía mínima garantizada por el Sistema Público Español de Pensiones incrementa en gran medida el coste de oportunidad de seguir trabajando al menos un año más, dado que retrasar la jubilación simplemente reduce el número de años que la persona recibirá la pensión de jubilación, sin incrementar esta pensión. Por lo tanto, la pensión mínima de jubilación incentiva a las personas a jubilarse tan pronto como sea posible recibir esta cuantía. Entre las personas que pueden beneficiarse de

\footnotetext{
${ }^{8}$ Dado el reducido número de observaciones, dentro de los beneficios de trabajar un año más, no tendremos en cuenta que a un trabajador le interese seguir trabajando porque su sueldo excede a la pensión de jubilación máxima.
} 
esta cuantía mínima se encuentran claramente los trabajadores con menor nivel de formación. Esto es así, como veremos más adelante, porque estos trabajadores reciben ingresos salariales bajos y que no varían mucho a lo largo de su vida laboral ${ }^{9}$.

Por último, el punto 3 puede ser un beneficio o un coste, lo cual dependerá del historial laboral pasado y presente de la persona. Específicamente, será un beneficio si los ingresos salariales que la persona espera recibir durante el corriente año son superiores a los ingresos salariales recibidos 8 o 15 años antes, dado que, de ser este el caso, aumenta la Base Reguladora. Pero el historial laboral pasado y presente de la persona, 0 dicho de otro modo, el perfil por edad de su renta salarial, depende de distintas variables, especialmente del nivel educativo de la persona. Como conclusión, entonces, debemos primero analizar el perfil por edad de la renta salarial, y determinar luego para qué grupos educativos este efecto es un beneficio o un coste.

\section{El perfil por edad de los ingresos salariales}

Para analizar los ingresos salariales durante la vida activa, hemos tomado los datos que reporta la Encuesta de Estructura Salarial del INE ${ }^{10}$. Esta encuesta muestra los salarios anuales de los trabajadores como función de diversas variables, como por ejemplo el sexo, el nivel educativo, la ocupación y la rama de actividad.

El Panel A de la Figura 1 muestra la renta salarial media anual de hombres y mujeres, para 9 grupos distintos de edad y 5 niveles educativos, en el año 199511. Como es de esperar, en el gráfico se observa que a mayor nivel educativo, mayor es la renta salarial media, especialmente después de los 25 años ${ }^{12}$.

Sin embargo, para una mejor visualización e interpretación de estos perfiles, procedemos seguidamente a aproximarlos a través de una función cuadrática ${ }^{13}$. Específicamente, postulamos que una persona con edad $j$ y nivel educativo $e$ tiene una renta salarial media anual, $w_{j, e}$, dada por la siguiente expresión:

$$
w_{j, e}=a_{0, e}+a_{1, e} j-a_{2, e} j^{2}
$$

\footnotetext{
${ }^{9}$ Las personas con pocos años cotizados a la Seguridad Social también se benefician de las pensiones mínimas de jubilación, pues en ese caso, su tasa de sutitución es baja, con lo cual su pensión de jubilación puede estar por debajo de la pensión mínima.

${ }^{10}$ Los datos están disponibles en: http://www.ine.es/inebmenu.

${ }^{11}$ La Encuesta de Estructura Salarial del Instituto Nacional de Estadística (INE) también aporta resultados detallados para el año 2002. Sin embargo, la muestra se divide en sólo cinco grupos por edad, de ahí que hayamos escogido la muestra del año 1995. Esta decisión, sin embargo, no afecta en absoluto a las conclusiones de este trabajo.

${ }^{12}$ No presentamos los datos diferenciados para hombres y mujeres, dado que la forma de estos perfiles son similares a través de los distintos grupos educativos.

${ }^{13}$ Utilizamos una aproximación cuadrática debido a la forma que toman los datos originales. Vemos que la aproximación se ajusta bastante a los datos reales.
} 
Los resultados derivados de aproximar cada uno de los 5 perfiles empíricos reportados en el Panel A de la Figura 1, se muestran en el Panel $B$ de la misma figura. El gráfico del Panel B permite apreciar dos hechos principales. Primero, si bien la renta salarial crece con la edad de la persona en todos los grupos educativos, las personas con menor nivel educativo tienen perfiles más planos durante su vida laboral. Y segundo, el valor máximo de la renta salarial media se observa a partir de la edad de 55. En algunos casos, el valor máximo se corresponde con el rango 55 a 59 años, (personas con estudios primarios, por ejemplo), mientras que en otros, esto sucede en el rango 60 a 65 (licenciados).

Figura 1.

Renta salarial bruta anual media (miles de euros).

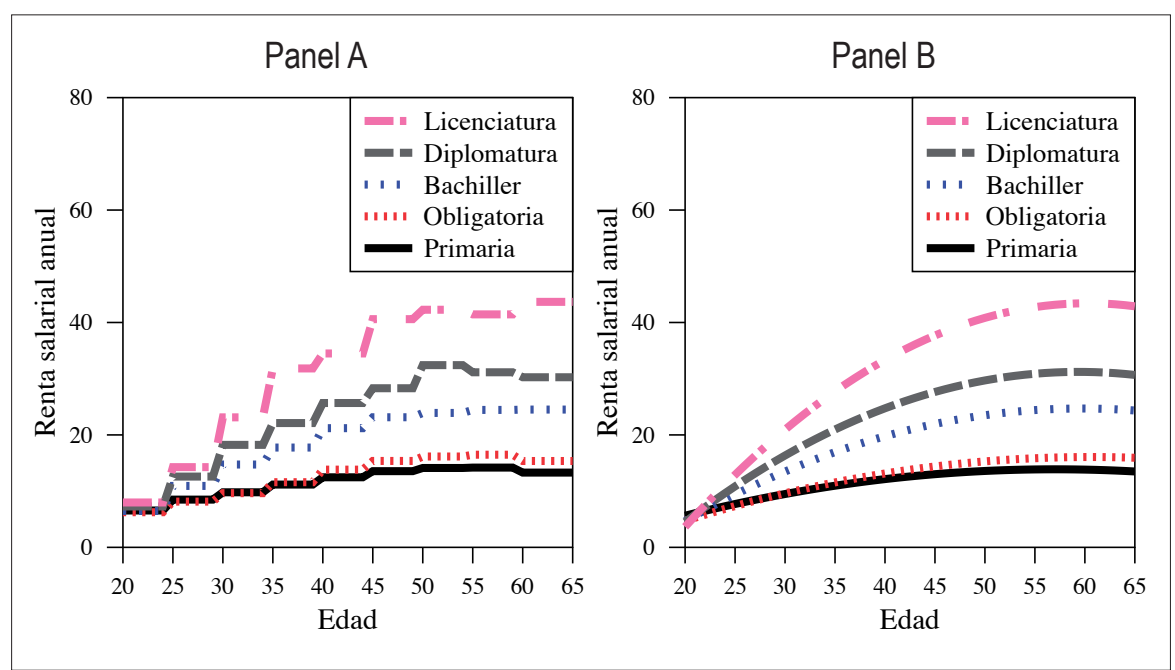

La Figura 1 nos permite analizar si el cambio en la Base Reguladora, en el caso de continuar trabajando un año más, puede ser considerado un coste 0 un beneficio. $O$ en otras palabras, si el hecho de trabajar un año más aumenta o disminuye la Base Reguladora. Consideremos primero un trabajador con el mayor nivel educativo, y cuya renta salarial se encuentra en la media de la distribución de su grupo educativo. Su perfil de renta salarial crece de forma sostenida con la edad, con lo cual los ingresos salariales que este trabajador medio espera recibir a los 60 y más son, sin duda, superiores a los observados 8015 años antes. Por lo tanto, si este trabajador decidiera trabajar un año más a los 60 años, su Base Reguladora crecería. Esto es un beneficio adicional derivado de la decisión de continuar trabajando. Lo mismo ocurre, aunque con menor intensidad, para trabajadores medios con estudios de Diplomatura y Bachiller, debido a que el crecimiento de la renta salarial con la edad es menor. 
Por otro lado, los trabajadores que están dentro de los dos grupos educativos más bajos, tienen un perfil de renta salarial muy plano. Esto implica que la renta salarial que esperan recibir si trabajan un año más después de los 60 no difiere en gran medida de la renta que han obtenido 8015 años antes, con lo cual, y en principio, no se modificaría la Base Reguladora. Sin embargo, conviene aclarar que a edades avanzadas son justamente los trabajadores con menor nivel educativo los que enfrentan mayores tasas de paro $^{14}$. En este caso, la cotización de una persona desempleada es, por lo general, el Salario Mínimo Interprofesional (SMI), cantidad inferior, por lo general, a la renta salarial bruta de un trabajador empleado con 45 años o más. Esto implica que si un trabajador está desempleado y decide no jubilarse, su Base Reguladora debe caer, dado que justamente su nueva base de cotización (SMI) es inferior a la base de cotización recibida 8 015 años antes.

Entonces, y como consecuencia de la evolución de la renta salarial durante el ciclo de vida, se puede concluir lo siguiente. Primero, los trabajadores con mayor educación, tienen un incentivo adicional para seguir trabajando después de los 60 años, dado que aumenta su Base Reguladora, aunque este incentivo puede disminuir a medida que el trabajador se acerca a los 65 años, debido a la concavidad en el perfil de ingresos salariales. Sin embargo, no se aprecia esto mismo para los individuos de menor educación; aquellos con educación primaria u obligatoria. Consecuentemente, estos individuos no tienen este incentivo, debido justamente a que su perfil de renta salarial es muy plano, con lo que trabajar un año más a partir de los 60 podría no aumentar su Base Reguladora. Pero incluso si una persona de uno de estos dos grupos educativos está desempleada, su Base Reguladora puede disminuir.

\section{La conducta óptima de jubilación}

En esta sección hemos señalado los beneficios y costes de seguir trabajando un año más para las personas con 60 años o más. También, hemos analizado el papel que desempeñan las pensiones mínimas de jubilación a la hora de decidir abandonar el mercado laboral. Y finalmente, hemos analizado los ingresos salariales por edad, dependiendo estos de la educación de los individuos. Con todo esto, podemos entonces describir cuál debería ser la conducta óptima de jubilación a partir de la primera edad legal de jubilación: 60 años.

\footnotetext{
${ }^{14}$ La tasa de paro para los trabajadores de 55 años o más, difiere notablemente con el nivel educativo alcanzado. De acuerdo a la Encuesta de Población Activa (EPA) realizada por el INE, en el segundo trimestre de 2008 la tasa de paro de los trabajadores con estudios primarios era del 8,3 por ciento; para los trabajadores con estudios de bachiller este número era 6,9 por ciento; y para los trabajadores con estudios universitarios, la tasa de paro era sólo 2,3 por ciento.
} 
A los 60 años

Dos importantes grupos de trabajadores deberían optar por jubilarse a esta edad. En primer lugar, es óptimo jubilarse para los trabajadores que se benefician de las pensiones mínimas de jubilación, debido a que ésta es la misma pensión que recibirán si eligen postergar su jubilación hasta los 65 años. Estos trabajadores se caracterizan por tener un bajo nivel de formación, y/o pocos años cotizados a la Seguridad Social. Y en segundo lugar, también deberían optar por abandonar el mercado laboral aquellos trabajadores que recibieran una muy baja base de cotización, en comparación con la que han estado recibiendo durante los años anteriores. Esto es debido a que si continúan trabajando un año más, su pensión caería debido a la caída en la Base Reguladora, y a pesar de que se reduce la penalización por jubilación anticipada. Esto último es el caso de trabajadores que han quedado desempleados, independientemente de su nivel educativo.

\section{Edades comprendidas entre los 61 y 64 años}

En este rango de edad no hay grandes incentivos para jubilarse. Esto se debe a que un trabajador puede beneficiarse si trabaja un año más, porque además de reducir la penalización por jubilación anticipada, podría también aumentar su tasa de sustitución. Además, si el trabajador espera recibir un salario alto durante el presente año, como sería el caso de los individuos con alto nivel educativo, su Base Reguladora aumenta. Sin embargo, a medida que aumenta la edad, los incentivos para continuar en el mercado laboral de un trabajador se reducen, a pesar de la reducción en la penalización por jubilación anticipada. Esto es una consecuencia directa del perfil cóncavo de las ganancias salariales de los trabajadores, como se muestra en la Figura 1.

\section{A los 65 o más años}

Los incentivos para abandonar el mercado laboral están en su nivel máximo. Esto se debe a dos motivos. En primer lugar y como ya hemos señalado, el perfil cóncavo de las ganancias salariales reduce la Base Reguladora. Segundo, el Sistema Público de Pensiones en España no proveía incentivos o premios, como el aumento de la pensión de jubilación, para aquellas personas que decidieran continuar trabajando pasados los 65 años de edad. Nótese que, y como ya se ha señalado, sólo en casos excepcionales un trabajador debería decidir continuar en el mercado laboral, como por ejemplo si esta persona esperara unos ingresos salariales suficientemente altos como para aumentar su Base Reguladora ${ }^{15}$.

\footnotetext{
${ }^{15}$ Especificamente, podría suceder que un trabajador con 65 o más años esperara recibir a esa edad un salario más alto que el que recibió 15 años antes. En este caso, su Base Reguladora aumenta, pues reemplaza un salario menor por uno mayor.
} 
Figura 2.

Probabilidades condicionadas de jubilación por edad (\%).
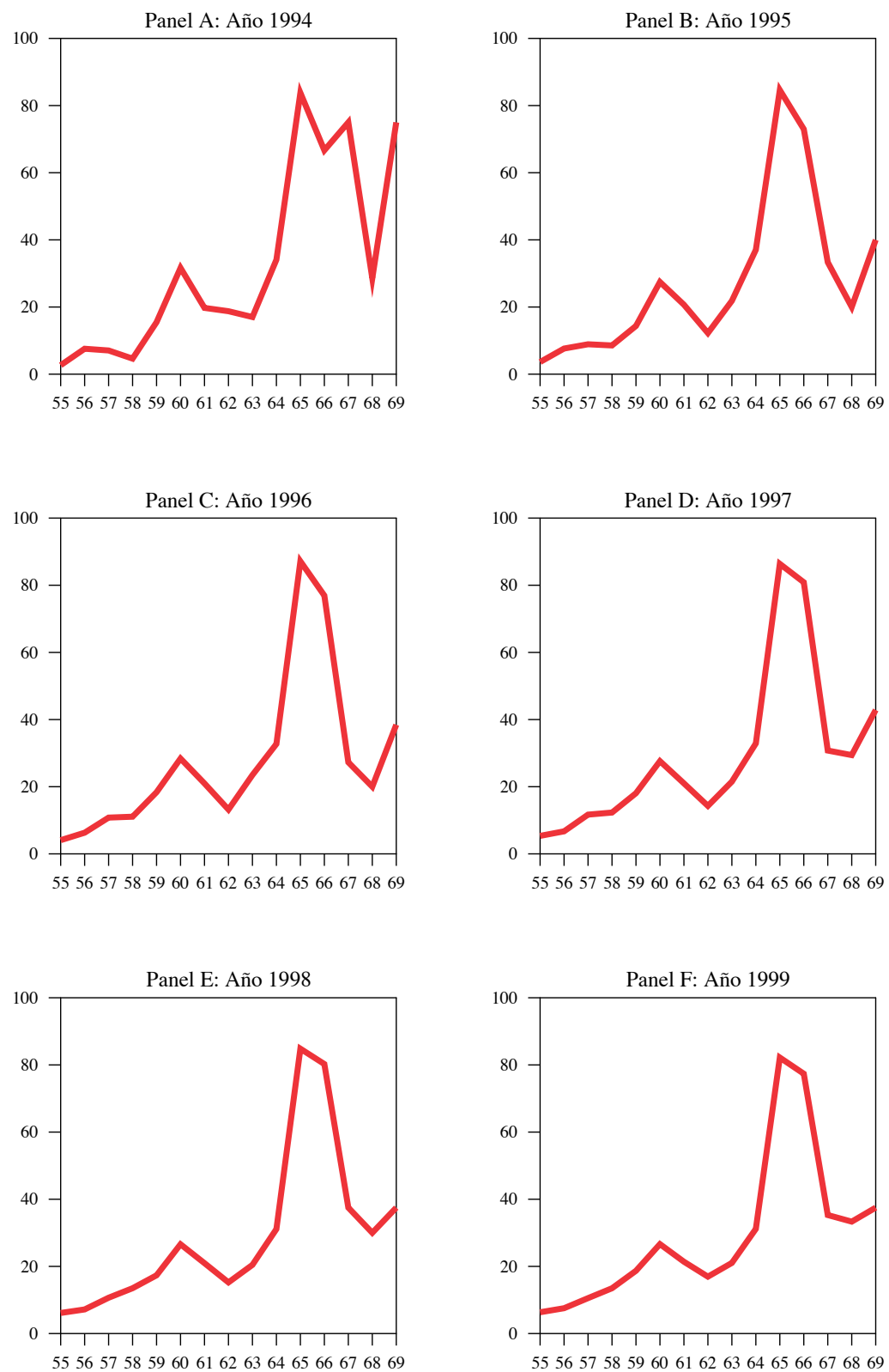

RIS, VOL.68. No 3, SEPTIEMBRE-DICIEMBRE, 775-795, 2010. ISSN: 0034-9712. DOI: 10.3989/ris.2008.10.29 
Si los trabajadores conocen y comprenden la normativa del sistema público de pensiones en España, la conducta empírica de jubilación debería ser similar a la conducta teórica de jubilación que acabamos de describir. Específicamente, los datos deberían mostrar los siguientes hechos:

1. Las tasas de abandono del mercado laboral muestran discontinuidades. Concretamente, hay dos tasas principales de abandono del mercado de trabajo: a los 60 y a los 65 años.

2. En el rango de 61 a 64 años de edad, las tasas de salida del mercado laboral aumentan, aunque son más bajas que las tasas observadas a los 60 y 65 años.

3. Después de los 65 años, la participación laboral es baja.

4. Cuanto mayor es el nivel educativo de la persona, más tarde se jubila.

\section{La evidencia empírica}

En esta sección, analizamos la evidencia empírica en España, respecto a la conducta de jubilación de los trabajadores, para ver si se corresponde con la conducta óptima de jubilación que ya hemos mencionado en la sección anterior. Este análisis lo hacemos en dos pasos. En primer lugar, procedemos a calcular las tasas de salida del mercado laboral. Y por último, analizamos las edades de jubilación y tasas de participación de los trabajadores, de acuerdo a su nivel educativo.

\section{Las tasas de salida del mercado laboral}

Para analizar las tasas de salida del mercado laboral en España, hacemos uso de los microdatos que tiene disponible el Panel de Hogares de la Unión Europea (ECHP), datos recolectados y difundidos por el INE. Esta encuesta, estudia el nivel de vida, las condiciones del mercado de trabajo y la cohesión social en relación con los requerimientos de información de las políticas activas de la Unión Europea en estos ámbitos y con sus efectos para la población. Las variables estudiadas, entre otras, son la situación económica (actual y en el año anterior), pobreza, empleo, actividad, migraciones laborales, jubilaciones, pensiones y nivel de formación. El panel abarca desde el año 1994 hasta el año 2001, ambos inclusive.

Por lo que respecta a la metodología, las variables que utilizamos son la situación económica actual y durante el año anterior a la entrevista de las personas con 55 a 69 años. Específicamente, tenemos en cuenta a todas las personas que el año anterior a la entrevista estaban en una situación activa, definida ésta como la situación en que una persona se encuentra dentro de uno de los dos siguientes grupos: trabajador o desempleado. Una vez identificada a la persona que está dentro de este grupo, analizamos cuál es su situación en el momento de la entrevista. En ese momento, la persona puede estar en una situación adicional: jubilada. Sea $A_{j t}$ el número de personas activas con edad $j$ al comienzo del año $t, y_{j t}$ el número de personas que se jubilan en esa misma 
edad y año. Entonces, podemos definir a la probabilidad condicional de jubilarse a la edad $j$ en el año $t$ (tasa de salida a la edad $j$ ), $S_{j t}$, como:

$$
S_{j t}=\left[\frac{J_{j t}}{A_{j t}}\right] \times 100
$$

Los resultados para el período 1994 a 2001 se muestran en la Figura 2. Como se puede apreciar, todos los gráficos, sin excepción alguna, muestran las discontinuidades a las que hacíamos referencia en el punto uno del apartado 3.3. Específicamente, las discontinuidades se producen en la primera edad legal de jubilación, 60 años y en la edad normal de jubilación, 65 años. ${ }^{16}$

También se observa en todos los gráficos que entre la edad 62 y la edad 64 las probabilidades condicionadas de jubilación aumentan. En el punto dos del apartado 3.3 llegamos a la conclusión de que esta misma tendencia debería ser observada a partir de la edad de 61 años. Por lo tanto, creemos que, y a pesar de esta discrepancia, los datos confirman nuestra conclusión. Por otra parte, la discrepancia puede deberse al número no muy alto de encuestados, lo cual también podría ser la razón para el comportamiento errático de estas probabilidades después de la edad de 66 años, en el año 1994.

Por último, analizamos las características de las personas que eligen jubilarse a los 60 y 65 años, para ver si estas características son consistentes con las que habíamos teorizado en nuestro análisis de la conducta óptima de jubilación. Específicamente, dijimos que en la primera edad de jubilación legal, es óptimo jubilarse para aquellos individuos con rentas salariales bajas o aquellos que recibirán la pensión mínima de jubilación. $Y$ a los 65 años, es óptimo jubilarse para todas aquellas personas que aún continuaban en la actividad, independientemente de su nivel educativo, dado que el sistema público de pensiones no otorgaba incentivos para seguir trabajando después de esa edad.

Los datos confirman nuestro análisis teórico. Por ejemplo, en el año 2000 y a la edad de 60 años, encontramos que el porcentaje de activos que, teniendo estudios primarios, de bachillerato, y de licenciatura, decidieron jubilarse fue 24,8; 11,1 y 4,3 por ciento respectivamente. Sin embargo, si miramos este mismo año pero para los activos con 65 años, estos números fueron 78,9; 90,0 y 44,4 por ciento respectivamente. Este mismo patrón se observa con mayor o menor similitud para el resto de los años de la encuesta.

\footnotetext{
${ }^{16}$ Antes de los 60 años, se observa que las probabilidades de jubilación son positivas, aunque menores al valor observado a los 60 . Esto es debido a que algunos regímenes especiales (Minería, por ejemplo) contemplan como la primera edad legal de jubilación a edades más tempranas que los 60 años.

Observar que la discontinuidad a los 60 años es mejor que a los 65 . Esto se debe a que realmente lo que estamos mostrando es la probabilidad condicionada de jubilación. A los 65 años el número de personas activas es menor, por lo tanto la probabilidad condicionada (suponiendo un número fijo de trabajadores jubilados) sería mayor.
} 
Por otra parte, no podemos observar en la muestra el número de personas que, cuando se jubilan, reciben la pensión mínima de jubilación. Pero de acuerdo con Sánchez-Martín (2001), en el año 1995 una gran mayoría (67,7 por ciento) de las personas que decidieron jubilarse a la edad de 60 años recibieron la pensión mínima de jubilación.

\section{Las tasas de actividad entre los 60 y 64 años}

Los últimos dos puntos que debemos corroborar tienen que ver con las edades medias de jubilación y las tasas de participación después de la edad de 65 años, por tipo educativo. Si bien el análisis realizado hasta ahora nos lleva a intuir que la evidencia empírica sí confirma nuestras conclusiones previas, en este apartado utilizamos la Encuesta de Población Activa (EPA), para ver si, efectivamente, las personas con mayor nivel educativo se jubilan a edades mayores, y que después de los 65 años, las tasas de participación son bajas, independientemente de grupo educativo considerado ${ }^{17}$.

La EPA no ofrece información directa acerca de las edades medias de jubilación por nivel de formación alcanzado. Sin embargo, estos números pueden ser observados indirectamente a través de las tasas de participación por tipo educativo en el rango 60 a 64 años. Específicamente, se puede concluir que a mayor tasa de actividad, mayor es la edad media de jubilación. Los datos referidos al período 1994 a 1999 se muestran en la Tabla $1^{18}$.

Tabla 1.

Tasas de participación por tipo educativo entre los 60 y 64 años.

\begin{tabular}{lcccccc}
\hline & 1994 & 1995 & 1996 & 1997 & 1998 & 1999 \\
\hline Primaria & 28,1 & 27,2 & 27,5 & 26,7 & 26,7 & 27,1 \\
Obligatoria & 34,6 & 30,2 & 31,4 & 35,5 & 34,5 & 30,7 \\
Bachiller & 37,4 & 40,7 & 35,3 & 35,5 & 33,4 & 34,7 \\
Diplomatura & 56,2 & 51,7 & 51,5 & 53,7 & 49,3 & 37,6 \\
Licenciatura & 73,5 & 67,7 & 67,8 & 63,3 & 68,8 & 64,3 \\
\hline
\end{tabular}

Fuente: INE. Los datos anuales corresponden al promedio de los cuatro trimestres.

\footnotetext{
${ }^{17}$ La EPA es una investigación por muestreo de periodicidad trimestral, realizada por el INE, dirigida a la población que reside en viviendas familiares del territorio nacional, y cuya finalidad es averiguar las características de dicha población en relación con el mercado de trabajo.

${ }^{18}$ Sólo se reportan los datos hasta el año 1999, debido a que a partir del año 2000 la clasificación de los grupos educativos cambia, y deja de ser directamente comparable a la que aquí hemos venido utilizando.
} 
Como puede verse claramente en la tabla 1, para todos los años se observa que cuanto mayor es el nivel de formación alcanzado, mayor es la tasa de participación. Esto implica a su vez que cuanto mayor es el nivel educativo, mayor es la edad media de jubilación, lo cual es consistente con nuestro análisis de la sección anterior. Específicamente, esto se debe a tres motivos. A mayor educación, menor es la probabilidad de recibir la pensión mínima, y por ende, de jubilarse anticipadamente. Pero también, cuanto mayor es la educación mayor es la renta salarial, y mayor es la probabilidad de que la persona incremente su Base Reguladora ${ }^{19}$. Finalmente, cuanto mayor es la educación, mayor es la renta salarial a la que se renuncia si se opta por la jubilación.

Tabla 2.

Tasas de participación por tipo educativo entre los 65 y 69 años (\%).

\begin{tabular}{lrrrrrr}
\hline & 1994 & 1995 & 1996 & 1997 & 1998 & 1999 \\
\hline Primaria & 3,8 & 4,4 & 3,5 & 2,9 & 3,0 & 3,3 \\
Obligatoria & 5,0 & 4,1 & 4,5 & 5,3 & 3,7 & 4,4 \\
Bachiller & 8,6 & 9,2 & 6,9 & 5,9 & 5,4 & 8,3 \\
Diplomatura & 11,1 & 8,1 & 9,8 & 6,4 & 5,5 & 9,2 \\
Licenciatura & 30,8 & 25,8 & 23,4 & 23,4 & 23,7 & 28,9 \\
\hline
\end{tabular}

Fuente: INE. Los datos anuales corresponden al promedio de los cuatro trimestres.

Tabla 3.

Tasas de participación por tipo educativo para 70 y más años (\%).

\begin{tabular}{lrrrrrr}
\hline & 1994 & 1995 & 1996 & 1997 & 1998 & 1999 \\
\hline Primaria & 0,7 & 0,7 & 0,5 & 0,3 & 0,5 & 0,5 \\
Obligatoria & 0,7 & 1,0 & 1,0 & 1,2 & 1,3 & 2,6 \\
Bachiller & 1,7 & 2,4 & 1,5 & 2,1 & 1,6 & 2,3 \\
Diplomatura & 1,9 & 2,0 & 1,7 & 1,4 & 1,6 & 0,8 \\
Licenciatura & 10,3 & 8,0 & 8,9 & 9,3 & 9,6 & 8,6 \\
\hline
\end{tabular}

Fuente: INE. Los datos anuales corresponden al promedio de los cuatro trimestres.

${ }^{19}$ Además, al trabajar un año adicional, reduce su penalización por jubilación anticipada. 
Por último, las Tablas 2 y 3 muestran las tasas de participación por tipo educativo, después de los 64 años, y para el período 1994 a 1999. Como se observa, los datos muestran que estas tasas son muy bajas para todos los tipos educativos, especialmente a partir de la edad de 70 años. Sin embargo, también las tasas de participación entre los 65 y 69 años son sustancialmente más bajas en comparación a las observadas para los mismos grupos educativos y el mismo período, pero en el rango de 60 a 64 años.

\section{OtRos FACTORES QUE AFECTAN A LA DECISIÓN DE LA JUBILACIÓN}

De la sección anterior concluimos que la conducta empírica de jubilación de los trabajadores en España está motivada por la normativa que rige el Sistema Público de Pensiones en España. Sin embargo, deberíamos matizar que esta normativa no es el único factor que afecta la decisión de jubilación. En esta sección describimos brevemente como el estado de salud del trabajador, su preferencia por el ocio o la pre-jubilación implementada por las empresas pueden condicionar o determinar también su conducta de jubilación.

Entre otras razones, la salud se deteriora, a medida que las personas envejecen. Por lo tanto, un trabajador con edad avanzada está más expuesto a padecer un empeoramiento repentino de su salud si lo comparamos con un trabajador más joven. Este deterioro en su estado de salud podría tener al menos dos implicaciones en lo que a su situación laboral se refiere. La primera es que el trabajador podría quedar imposibilitado para continuar trabajando, lo cual forzaría su jubilación. Y la segunda es que a pesar de poder continuar trabajando, el trabajador decidiese abandonar el mercado laboral con el objeto de no empeorar su salud ${ }^{20}$. Estas implicaciones han sido demostradas en estudios como los de Bound et al. (1999), Au et al. (2005) y Disney et al. (2006).

Por lo general, los individuos prefieren el ocio (o tiempo libre) a trabajar. Sin embargo, la preferencia por el ocio puede cambiar con la edad del trabajador. Específicamente, puede ser que, a medida que el trabajador envejece, éste otorgue un mayor valor a cada hora de ocio de la que disponga, en comparación con un trabajador más joven. Esto por lo tanto implica que a medida que el trabajador envejece, sus incentivos a jubilarse aumentan, para poder disfrutar así de un mayor tiempo de ocio. Este argumento ha sido demostrado por autores como Gordon y Blinder (1980) y Gustman y Steinmeier (1986).

La prejubilación de un trabajador consiste en un acuerdo entre el trabajador y la empresa por el que se deja de trabajar y se recibe una indemnización, una asignación social y un complemento por parte de la empresa. Por lo general, este acuerdo implica que el trabajador pasa a ser una persona inactiva, y los motivos que pueden desem-

${ }^{20}$ También, y a pesar de poder seguir trabajando, el trabajador podría decidir abandonar el empleo, dado que los sistemas de Seguridad Social proveen pensiones por incapacidad, las cuales son un camino alternativo hacia la jubilación. 
bocar en este acuerdo son, entre otros, una reconversión industrial, o una situación de crisis financiera en la respectiva empresa. En España las prejubilaciones han sido frecuentes durante las últimas décadas, especialmente durante los períodos de bajo o nulo crecimiento económico y afectan principalmente a los trabajadores con edades comprendidas entre los 50 y 65 años.

Los factores que acabamos de describir se reflejan también en los datos que describen la conducta empírica de la jubilación de los trabajadores en España. Por ejemplo, las bajas tasas de participación laboral después de los 65 años, no sólo muestran los pocos incentivos a continuar trabajando que otorga el Sistema Público de Pensiones, sino también que en parte se deben a factores como la salud, la preferencia por el ocio, o las prejubilaciones por parte de las empresas. Como conclusión, entonces, podemos afirmar que además de los incentivos que facilita el Sistema Público de Pensiones a través de su normativa, y que es conocida y comprendida por los trabajadores, la decisión de jubilarse de un trabajador también se ve afectada por las circunstancias mencionadas anteriormente.

\section{CONCLUSIONES}

Este trabajo intenta responder a la pregunta de si los trabajadores en España conocen y comprenden la normativa que rige el Sistema Público Español de Pensiones. Para responder a esta pregunta realizamos el siguiente análisis: primero, analizamos la normativa del principal régimen que compone el sistema de pensiones en España, el Régimen General de la Seguridad Social. Posteriormente, y según dichas normas, obtenemos una conducta teórica óptima de jubilación, dadas las características individuales del trabajador. Por último, comparamos esta conducta con la conducta empírica de jubilación de los trabajadores en España. Nuestros resultados muestran que ambas conductas son similares, con lo cual concluimos que los trabajadores con edad avanzada sí conocen y comprenden las normas que establece el Régimen General de la Seguridad Social.

Nuestros resultados no son completamente incompatibles con los trabajos que afirman que los trabajadores no conocen la normativa de jubilación. Una posible explicación que concilia las dos visiones es la siguiente: nuestros resultados, sumados a aquellos obtenidos por otros autores para otros países, confirmarían que los trabajadores con edades avanzadas sí comprenden la normativa. Pero muy posiblemente, los trabajadores más jóvenes desconocen la normativa del sistema de pensiones. Esto de debería a que un trabajador joven percibe su jubilación como un evento muy lejano en el tiempo, y conocer y comprender esta normativa le supone un coste adicional. A medida que el trabajador envejece su jubilación se aproxima en el tiempo, lo que le incentiva a dedicar tiempo para informarse y analizar cuál es el momento óptimo para abandonar el mercado laboral. Sin embargo, esta es sólo una hipótesis, que creemos, debería ser investigada en profundidad. 
Las conclusiones obtenidas en este trabajo tienen implicaciones importantes a la hora de la elección de las políticas óptimas que mejoren las sostenibilidad del sistema de la Seguridad Social. Los estudios económicos acerca de las consecuencias para la viabilidad financiera futura de distintas reformas de los sistemas de pensiones, salvo pocas excepciones, asumen que los trabajadores desconocen la normativa de los sistemas de pensiones. Este supuesto, sin embargo, parece no corresponderse con la realidad. Los resultados aportados en este artículo demuestran que si se cambia la normativa del sistema de pensiones, los trabajadores cambiarán su conducta de jubilación.

Partiendo de la situación previa en la que los trabajadores van a reaccionar a cambios en la normativa, el siguiente paso será buscar cuál es la normativa óptima dados los objetivos establecidos. Por lo tanto aparecen dos nuevas cuestiones que quedan fuera del alcance de este artículo pero que deberían ser estudiadas. Por un lado habrá que definir cuáles son los objetivos que se están buscando y por otro lado habrá que ver cuáles son los incentivos de los trabajadores, dado que, conociendo estos incentivos se podrán establecer las reformas más efectivas para conseguir los objetivos establecidos.

\section{REFERENCIAS BIBLIOGRÁFICAS}

Au, D., T. Crossley y M. Schellhorn. 2005. "The Effects of Health Shocks and Long-term Health on the Work Activity of Older Canadians." Health Economics 14: 999-1018.

Boldrin, M. y S. Jimenez-Martín. 2002. "Evaluating Spanish Pension Expenditure Under Alternative Reform Scenarios." Pp. 351-412 in Social Security and Retirement Across The World: Fiscal Implications, coordinated by J. Gruber y D. Wise. Chicago: Chicago University Press.

Börsch-Supan, A., Kohnz, S. y R. Schnabel. 2002. "Micro Modeling of Retirement: Decisions in Germany." Discussion paper 020, Mannheim Research Institute for the Economics of Aging (MEA).

Bound, J., M. Schoenbaum, T. Stinebrickner y T. Waidmann. 1999. "The Dynamic Effects of Health on the Labor Force Transitions of Older Workers." Labour Economics 6: 179-202.

Disney, R., C. Emmerson y M. Wakefield. 2006. "Ill Health and Retirement in Britain: A Panel Databased Analysis." Journal of Health Economics 25: 621-649.

Fatas, E., Lacomba, J. y Lagos, F. 2007. "An Experimental Test on Retirement Decisions." Economic Inquiry 45: 602-614.

Feldstein, M. S. 1977. "Social Security and Private Savings: International Evidence in and Extended Lyfe-Cycle Model." Pp. 187-209 in The Economics of Public Services, Proceedings of a Conference held by the International Economics Association.

Gordon, R. H., y A. Blinder. 1980. "Market Wages, Reservation Wages and Retirement." Journal of Public Economics 14: 277-308.

Gustman, A. y T. Steinmeier. 1986. "A structural retirement model.” Econometrica 54: 555-584. 
Juster, F. T. y R. Suzman. 1995. "An Overview of the Health and Retirement Study." Journal of Human Resources 30: S7-S56.

Kotlikoff, L. J. 1979. "Social Security and equilibrium Capital Intensity." Quarterly Journal of Economics 93: 233-253.

Lacomba, J. y F. Lagos. 2007. "Political election on legal retirement age." Social Choice and Welfare 29: 1-17.

Mahieu R. and D. Blanchet. 2004. "Estimating Models of Retirement Behavior on French Data." Pp: 235-284 en Social Security Programs and Retirement Around The World, coordinated by J. Gruber and D. Wise. Chicago: The University of Chicago Press.

Mitchell, O. S. 1988. "Worker Knowledge of Pension Provisions." Journal of Labor Economics 6: 21-39.

Sánchez-Martín A. 2001. "Endogenous Retirement And Public Pension System Reform In Spain." Economics Working Papers we013503, Universidad Carlos III, Departamento de Economía. Madrid.

Sheshinski, E. 1978. "A Model of Social Security and Retirement Decisions." Journal of Public Economics 10: $337-360$.

Wise, D. 1997. "Retirement Against The Demographic Trend: More Older People Living Longer, Working Less, And Saving Less." Demography 34: 83-95.

RAMÓN COBO-REYES es Doctor en Economía por la Universidad de Granada. Su investigación se ha centrado en el ámbito de la economía experimental y del comportamiento con especial atención a las redes sociales. Actualmente es profesor en el Departamento de Teoría e Historia Económica de la Universidad de Granada.

JULIÁN DÍAZ-SAAVEDRA es Doctor en Economía por la Universidad Carlos III de Madrid. Su investigación se ha centrado en el estudio de la sostenibilidad financiera de los sistemas públicos de pensiones, así como en las consecuencias agregadas, distributivas y de bienestar, de reformas paramétricas diseñadas para mejorar la sostenibilidad de dichos sistemas. Actualmente es profesor en el Departamento de Teoría e Historia Económica de la Universidad de Granada.

RECIBIDO: 29/10/2008

ACEPTADO: 26/05/2009 